and Mycobacterium goodii sp. nov., two new rapidly growing species related to Mycobacterium smegmatis and associated with human wound infections: a cooperative study from the International Working Group on Mycobacterial Taxonomy. Int J Syst Bacteriol. 1999;49:1493-511. https:/ / doi.org/10.1099/00207713-49-4-1493

6. Salas NM, Klein N. Mycobacterium goodii: an emerging nosocomial pathogen: a case report and review of the literature. Infect Dis Clin Pract (Baltim Md). 2017;25:62-5. https://doi.org/10.1097/IPC.0000000000000428

7. Harper G, Ong JL, Costanigro L. Oh Goody! Two additional Mycobacterium goodii infections. Open Forum Infectious Diseases. 2015;2(suppl_1):576. https://doi.org/10.1093/ ofid/ofv133.450

8. Parikh RB, Grant M. Mycobacterium goodii endocarditis following mitral valve ring annuloplasty. Ann Clin Microbiol Antimicrob. 2017;16:14. https:/ / doi.org/10.1186/s12941-0170190-4

9. Goussard P, Rabie H, Morrison J, Schubert PT. Superinfection with Mycobacteria goodii in a young infant with exogenous lipoid pneumonia. Pediatr Pulmonol. 2019;54:1345-7. https://doi.org/10.1002/ppul.24355

10. Hougas JE III, Bruneteau RJ, Varman M. Mycobacterium goodii infection of skin graft in an immunocompetent child. Infect Dis Clin Pract. 2011;19:146-7. https:/ / doi.org/10.1097/ IPC.0b013e3182002df1

Address for correspondence: Christopher Ouellette, Division of Infectious Diseases and Host Defense Program, Nationwide Children's Hospital, 700 Children's Dr, Rm C5C-J5432, Columbus, OH 43205, USA; email: christopher.ouellette@ nationwidechildrens.org

\section{Sporotrichosis Cases in Commercial Insurance Data, United States, 2012-2018}

\author{
Kaitlin Benedict, Brendan R. Jackson \\ Author affiliation: Centers for Disease Control and Prevention, \\ Atlanta, Georgia, USA
}

DOI: https://doi.org/10.3201/eid2611.201693

The geographic distribution of sporotrichosis in the United States is largely unknown. In a large commercial health insurance database, sporotrichosis was rare but most frequently occurred in southern and south-central states. Knowledge about where sporotrichosis is most likely to occur is essential for increasing clinician awareness of this rare fungal disease.
Sporotrichosis is an infection caused by the fungus Sporothrix. The infection typically follows cutaneous inoculation and involves the skin, subcutaneous tissue, and lymph nodes; pulmonary or disseminated disease occurs less frequently and usually affects immunocompromised persons (1). Sporothrix exists nearly worldwide in soil and decaying plant matter, but many unanswered questions remain about its precise ecologic niche (2). In the United States, its geographic distribution is poorly understood. Knowledge about where sporotrichosis is most likely to occur can help healthcare providers recognize and treat it earlier and help public health officials focus prevention messages.

We used the MarketScan Research Databases (IBM, https://www.ibm.com) to examine the geographic distribution of sporotrichosis in the United States. These databases comprise health insurance claims data from outpatient visits, prescriptions, and hospitalizations for employees, dependents, and retirees throughout the United States. In 2018, the databases contained records for $\approx 27$ million persons. MarketScan data are fully de-identified; thus, the Centers for Disease Control and Prevention institutional review board did not need to approve this study.

To query the database, we used Treatment Pathways (IBM), a web-based platform, that comprises data from persons with health insurance plans that contributed prescription drug information to the MarketScan databases. We used data from February 1, 2012-December 31, 2018, to identify sporotrichosis patients using code 117.1 from the International Classification of Diseases (ICD), Ninth Revision, Clinical Modification and code B42 from the ICD, 10th Revision, Clinical Modification (ICD-10-CM). We used the primary beneficiary's state of residence to calculate average annual state-specific rates per 1 million MarketScan enrollees. We evaluated underlying conditions on or in the month before sporotrichosis diagnosis, demographic features, and type of sporotrichosis.

Of $\approx 76$ million unique patients during 2012-2018, 1,322 had a sporotrichosis diagnosis code. For 1,236 $(93.5 \%)$ of those, information was available about state of residence. The average annual rate of sporotrichosis cases per 1 million enrollees was highest in Oklahoma (6.1), Michigan (3.9), Kansas (3.5), and Kentucky (3.5) (Figure). Nationwide, the average annual rate was 2.0 cases/1 million enrollees.

For the 1,252 patients continuously enrolled during the month before their diagnosis, median age was 54 years; most (62\%) patients were female (Table). The most common underlying conditions we evaluated were diabetes $(7 \%)$, immune-mediated inflammatory disease $(3 \%)$, and chronic obstructive pulmonary 
disease (2\%). Among 514 patients with sporotrichosis ICD-10-CM codes, specific types included lymphocutaneous in $13 \%$ and unspecified forms in $69 \%$. Thirtyseven $(3 \%)$ patients were hospitalized at diagnosis.

Although sporotrichosis occurred rarely in this large sample of privately insured patients, it was most common in the southern and south-central United States. The US geographic distribution of sporotrichosis has not been well described since the 1940s, when most cases were observed in the Mississippi River basin, with the highest frequencies in North Dakota, Nebraska, Wisconsin, Kansas, and Missouri (3). Reasons for the low rates we found in North Dakota and Wisconsin and the high rates in Michigan are unknown. Historically, Wisconsin-grown sphagnum moss has been the most common source of sporotrichosis outbreaks. At least 8 published outbreaks implicated Wisconsin moss shipped to other states for use in topiaries or packing material for tree seedlings $(4,5)$. Since the late 1990s, sporotrichosis outbreaks appeared absent from published literature (4). Industry changes in harvesting sources or processing methods might play a role in the absence of outbreaks (5). Public health officials might not detect or investigate these outbreaks, as no routine surveillance for sporotrichosis exists. Our observation of higher sporotrichosis rates in Oklahoma and Kansas is consistent with past outbreaks linked to contaminated hay in those states (6-8).

In our analysis, sporotrichosis more frequently affected older women. These results possibly reflected differences in care-seeking behavior or exposures.
Underlying conditions were uncommon, suggesting that most cases occurred in previously healthy persons. This finding was consistent with lymphocutaneous disease resulting from traumatic inoculation.

Our findings are subject to several limitations. The primary limitation was that patients' states of residence might not represent the exposure location or the original environmental source of Sporothrix. Undetected cases and potential case misclassification are inherent limitations of administrative data. Diagnosis codes might substantially underestimate the true number of cases because affected persons might not seek care for self-limiting sporotrichosis. In addition, sporotrichosis might not have been correctly diagnosed or coded. Furthermore, administrative data often lack detail (i.e., differentiating between different sporotrichosis forms is not possible in ICD's Ninth Revision, Clinical Modification, and was not frequently used in ICD-10-CM). However, MarketScan is one of the few data sources large enough to provide a sufficient number of sporotrichosis cases to analyze at a subregional level.

Knowledge about where sporotrichosis is most likely to occur is essential for increasing clinician awareness of this rare disease. Increased awareness might lead to faster diagnosis and treatment with antifungal medication, which most sporotrichosis patients need (1). Understanding the distribution of sporotrichosis is also essential for understanding emerging sources of infection. Parts of Latin America are experiencing a large and growing epidemic of severe sporotrichosis caused by cat-transmitted

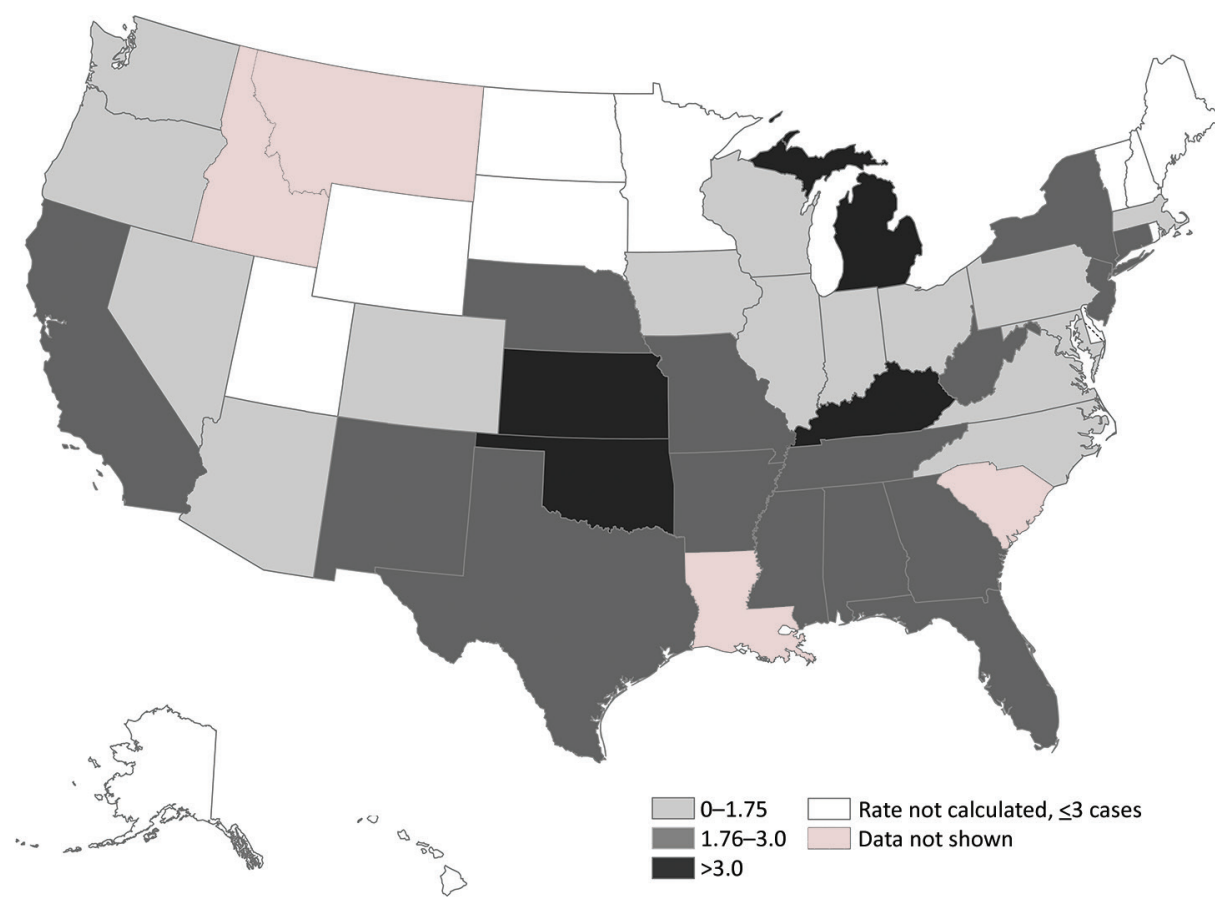

Figure. Average annual sporotrichosis rate per 1 million MarketScan enrollees, United States, 2012-2018. MarketScan (IBM, https://www.ibm.com) data are a subset of privately insured persons in the United States and are not necessarily representative of persons with other types of health insurance or of persons without insurance. To avoid potentially unreliable estimates, the rates for states with $\leq 3$ cases were not calculated. MarketScan does not permit state-level analyses for certain states. 
Table. Characteristics of 1,252 patients with sporotrichosis, United States, 2012-2018*

\begin{tabular}{|c|c|}
\hline Characteristic & No. (\%) \\
\hline \multicolumn{2}{|l|}{ Age group, y† } \\
\hline $0-17$ & $46(4)$ \\
\hline $18-34$ & $153(12)$ \\
\hline $35-44$ & $154(12)$ \\
\hline $45-54$ & $305(24)$ \\
\hline $55-64$ & $402(32)$ \\
\hline$\geq 65$ & $192(15)$ \\
\hline \multicolumn{2}{|l|}{$\overline{\operatorname{Sex}}$} \\
\hline $\mathrm{F}$ & $774(62)$ \\
\hline M & $478(38)$ \\
\hline Residence in a rural area & $184(15)$ \\
\hline \multicolumn{2}{|l|}{ Type of sporotrichosis } \\
\hline Lymphocutaneous $\ddagger$ & $68(13)$ \\
\hline Pulmonary§ & $27(5)$ \\
\hline Other forms & $71(14)$ \\
\hline Unspecified\# & 357 (69) \\
\hline \multicolumn{2}{|l|}{$\begin{array}{l}\text { Underlying conditions on or in the month before } \\
\text { sporotrichosis diagnosis }\end{array}$} \\
\hline Alcohol related disorders ${ }^{* *}$ & $9(0.7)$ \\
\hline Immune-mediated inflammatory disease †† & $39(3)$ \\
\hline Chronic obstructive pulmonary disease $\ddagger \ddagger$ & $26(2)$ \\
\hline Diabetes§§ & $90(7)$ \\
\hline HIV diseasef & $5(0.4)$ \\
\hline Solid organ or stem cell transplant\#\# & $5(0.4)$ \\
\hline Hematologic malignancy ${ }^{* * *}$ & $7(0.6)$ \\
\hline \multicolumn{2}{|c|}{ 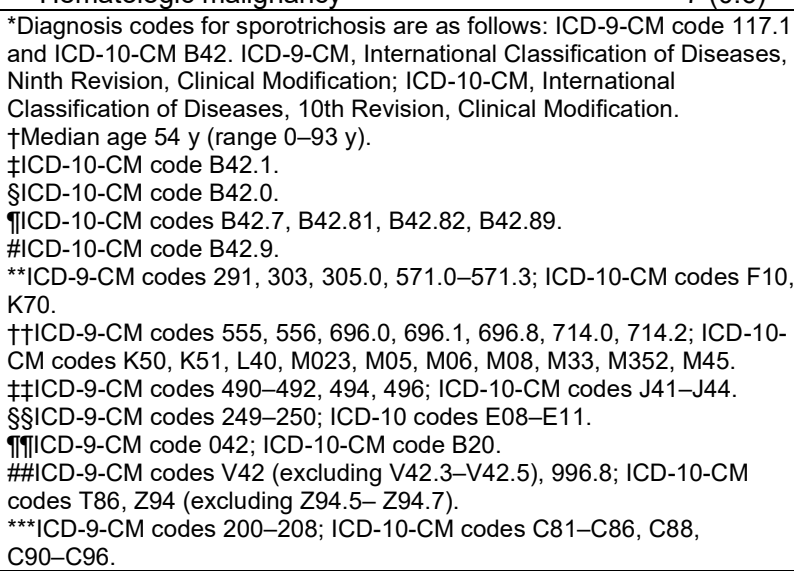 } \\
\hline
\end{tabular}

S. brasiliensis (9). This emerging infection provides further evidence of the need for ongoing monitoring.

\section{Acknowledgments}

We thank Lance Owen for assistance with the Figure and John Rossow for subject matter expertise.

\section{About the Author}

Ms. Benedict is an epidemiologist in the Mycotic Diseases Branch, Division of Foodborne, Waterborne, and Environmental Diseases, National Center for Emerging and Zoonotic Infectious Diseases, CDC. Her research interests include the epidemiology and prevention of fungal infections.

\section{References}

1. Kauffman CA, Bustamante B, Chapman SW, Pappas PG; Infectious Diseases Society of America. Clinical practice guidelines for the management of sporotrichosis: 2007 update by the Infectious Diseases Society of America. Clin Infect Dis. 2007;45:1255-65. https://doi.org/10.1086/522765

2. Chakrabarti A, Bonifaz A, Gutierrez-Galhardo MC, Mochizuki T, Li S. Global epidemiology of sporotrichosis. Med Mycol. 2015;53:3-14. https://doi.org/10.1093/mmy/ myu062

3. Gastineau F, Spolyar L, Haynes E. Sporotrichosis: report of six cases among florists. J Am Med Assoc. 1941;117:1074-7. https:/ / doi.org/10.1001/jama.1941.02820390016005

4. Hajjeh R, McDonnell S, Reef S, Licitra C, Hankins M, Toth B, et al. Outbreak of sporotrichosis among tree nursery workers. J Infect Dis. 1997;176:499-504. https:/ / doi.org/10.1086/514070

5. Coles FB, Schuchat A, Hibbs JR, Kondracki SF, Salkin IF, Dixon DM, et al. A multistate outbreak of sporotrichosis associated with sphagnum moss. Am J Epidemiol. 1992;136:475-87. https:/ / doi.org/10.1093/oxfordjournals.aje. a116521

6. Dooley DP, Bostic PS, Beckius ML. Spook house sporotrichosis. A point-source outbreak of sporotrichosis associated with hay bale props in a Halloween hauntedhouse. Arch Intern Med. 1997;157:1885-7. https:/ / doi.org/ 10.1001/archinte.1997.00440370135014

7. Centers for Disease Control (CDC). Sporotrichosis among hay-mulching workers - Oklahoma, New Mexico. MMWR Morb Mortal Wkly Rep. 1984;33:682-3.

8. Dahl BA, Silberfarb PM, Sarosi GA, Weeks RJ, Tosh FE. Sporotrichosis in children. Report of an epidemic. JAMA. 1971;215:1980-2. https://doi.org/10.1001/ jama.1971.03180250072022

9. Gremião IDF, Oliveira MME, Monteiro de Miranda LH, Saraiva Freitas DF, Pereira SA. Geographic expansion of sporotrichosis, Brazil. Emerg Infect Dis. 2020;26:621-4. https://doi.org/10.3201/eid2603.190803

Address for correspondence: Kaitlin Benedict, Centers for Disease Control and Prevention, 1600 Clifton Rd NE, Mailstop H24-9, Atlanta, GA 30329-4027, USA; email: jsy8@cdc.gov 